\title{
Anterior cruciate ligament reconstruction and rehabilitation: predictors of functional outcome
}

\author{
FRANCESCO DELLA VILLA ${ }^{1}$, MARGHERITA RICCI ${ }^{1}$, FRANCESCO PERDISA ${ }^{2}$, \\ GIUSEPPE FILARDO ${ }^{2}$, JACOPO GAMBERINI ${ }^{1}$, DANIELE CAMINATI ${ }^{1}$, STEFANO DELLA VILLA ${ }^{1}$ \\ ${ }^{1}$ Isokinetic Medical Group, FIFA Medical Centre of Excellence, Bologna, Italy \\ 2 Rizzoli Orthopaedic Institute, 2nd Clinic - Biomechanics Laboratory, Bologna, Italy
}

\begin{abstract}
Surgical reconstruction of an injured anterior cruciate ligament (ACL) leads to full recovery of function and sports activity in a high percentage of cases. The aim of the present study was to analyze variables related to the patient, the surgical technique and the post-surgical rehabilitation methods, seeking to identify predictors of outcome and recovery time after ACL reconstruction. One hundred and four patients $(81 \mathrm{M}, 23 \mathrm{~F})$ undergoing a step-based rehabilitation protocol after ACL reconstruction were evaluated. $43.2 \%$ of them had an isolated ACL lesion, whereas $56.8 \%$ had one or more concurrent injuries. Data relating to personal characteristics, surgery and post-operative management were collected and analyzed for correlation. Clinical outcome was evaluated with IKDC subjective score and the Tegner score, and the time to reach full recovery was noted as well.

Young patients with a higher pre-injury Tegner activity level or who practice sport at professional level, no concurrent capsular lesions and no postoperative knee bracing had better clinical results and took shorter time to recover. Also, a higher percentage of on-thefield rehabilitation sessions, and absence of significant muscle strength deficits at the first knee isokinetic test emerged as rehabilitation-related factors leading to a better post-surgical outcome.

Personal, surgical and rehabilitation factors should be
\end{abstract}

\section{Corresponding Author:}

Francesco Della Villa, MD

Isokinetic Medical Group, FIFA Medical Centre of Excellence

Bologna, Italy

E-mail: f.dellavilla@isokinetic.com considered in order to optimize patient management and maximize the expected results. Further studies are needed to find the strongest factors in different patients. Level of evidence: Level IV, retrospective study.

Keywords: ACL reconstruction, rehabilitation, knee, functional outcome.

\section{Introduction}

Anterior cruciate ligament (ACL) lesions may lead to knee instability, thus seriously restricting patients' return to sports activity and jeopardizing joint homeostasis, with deleterious long-term results (1). Surgical reconstruction is the gold standard for the management of ACL ruptures in a sports-active population, showing a high rate of success in terms of recovery of normal knee function and return to the previous level of sports activity (2). In addition to several considerations regarding different kinds of graft and techniques available for reconstruction, clinical research has recently been focusing on the critical role of patientrelated factors, which might influence the final results and the time needed for recovery. In fact, different patient- and injury-related factors might be associated with a better outcome and an earlier return to the preinjury level of sports activity. Among these, postoperative rehabilitation has been acknowledged to play a key role. However, a recent systematic review reported contradictory findings regarding return to sport and a lack of consensus on the most appropriate timing and predictors to consider in order to allow athletes to resume their previous level of activity in safety (3). The objective findings most commonly sought before 
allowing unrestricted sports activity are: a negative Lachman test, a complete range of motion (ROM) and a negative pivot shift test, whereas a relatively small number of clinicians consider muscle strength analysis important (4).

Table 1. All quantitative variables of the patients, expressed as mean, standard deviation and range.

\begin{tabular}{ll}
\hline Variables & Mean \pm SD (range) \\
\hline Age & $28.4 \pm 11.1$ years $(14-59)$ \\
Weight & $74.0 \pm 10.5 \mathrm{~kg}(47-106)$ \\
Tegner score pre-injury & $7.6 \pm 1.7(3-10)$ \\
Surgery-to-rehabilitation time (days) & $8.6 \pm 6.0(1-70)$ \\
Pain (NRS 1-10) & $3.7 \pm 2.2(0-9)$ \\
Knee maximum flexion angle & $73.5^{\circ} \pm 23.1^{\circ}(5-130)$ \\
Knee extension deficit & $1.5^{\circ} \pm 3.7^{\circ}\left(0-15^{\circ}\right)$ \\
Knee effusion $(0-4)$ & $1.5 \pm 0.6(1-3)$ \\
\hline
\end{tabular}

A better understanding of the relationship between patient-related factors, rehabilitation and final outcome might be useful in order to develop more efficient pre- and postoperative training protocols, and thus optimize the expected postoperative outcomes. The aim of this study was therefore to identify patientrelated and rehabilitation-related factors as predictors of functional outcome after ACL reconstruction (ACLR), to allow tailored postoperative care algorithms and better functional outcomes.

\section{Methods}

\section{Patient selection}

Data from 104 consecutive patients (81 men and 23 women) who underwent rehabilitation after ACLR in a single center were collected from the medical charts. They all followed a specific criteria-based rehabilitation protocol, which was functionally oriented and tailored to each patient.

To be included, patients had to have: i) undergone an ACLR procedure, and ii) followed the post-surgical rehabilitation program in the same rehabilitation center. Ninety-five patients, evaluated at a minimum follow-up of 12 months after surgery, were included in the analysis, whereas nine who, for geographical or economic reasons, stopped attending the rehabilitation center prior to the discharge time were excluded. Of the 95 patients evaluated at 12 months of follow-up, 87 were competitive (lower divisions) or recreational athletes; the other eight were professional athletes. The majority of the patients $(78 \%)$ had a non-contact ACL injury, whereas $19 \%$ had sustained a contact ACL injury and the remaining 3\% had been involved in a car accident. An isolated ACL lesion was detected in 41 cases (43.2\%), whereas the remaining 54 patients $(56.8 \%)$ had at least one concomitant combined lesion. All but 13 cases $(13.7 \%)$ had undergone primary ACLR. For the reconstruction, a hamstring autograft (semitendinosus and gracilis tendons) was used in 82 cases (86.3\%), an allograft in nine cases $(9.5 \%)$, and a patellar tendon autograft in the remaining four patients $(4.2 \%)$.

The mean time between surgery and the beginning of rehabilitation was $8.6 \pm 6$ days (1-70). At the first postoperative clinical consultation, $43 \%$ of the patients wore a knee brace. All other quantitative variables are listed in Table 1.

\section{Rehabilitation strategy}

A teamwork-based strategy was applied: rehabilitation specialists were coordinated by a sports physician, responsible for periodically checking the patient's progression through the rehabilitation process. We adopted a functionally-oriented, criteria-based rehabilitation protocol, which was customized for each patient and included a mixture of supervised gymnasium, pool and on-field rehabilitation (OFR) sessions.

The protocol is graduated and pursues four functional goals: 1) walking without crutches; 2) running on a treadmill; 3) starting OFR; and 4) return to professional or competitive/recreational sports activity.

Progression from one stage to the next was based on safety criteria and not time. Patients meeting all the criteria for a specific goal could proceed to the next stage in the rehabilitation protocol. The criteria for meeting the first goal were: a) no surgical contraindications; b) absent or minimal knee effusion; c) full knee extension (comparable to contralateral knee extension); and d) recovery of the normal gait cycle. The criteria for attainment of the second goal were: a) absent or minimal pain on a numerical rating scale (NRS) (NRS <3) during walking; b) knee flexion $>120^{\circ}$; and c) proper trunk, thigh and leg muscle tone. For the third goal they were: a) knee extensor and flexor strength $80 \%$ compared to the contralateral limb, as evaluated using the knee isokinetic test (IT); and b) being able to run on a treadmill at $8 \mathrm{~km} / \mathrm{h}$ at least for 
10'. For the fourth goal they were: a) surgeon consensus; b) knee extensor and flexor strength 100\% (as evaluated using the IT); c) appropriate recovery of endurance (as evaluated using the lactate aerobic-anaerobic threshold test); and d) completion of the OFR rehabilitation program (5).

\section{Patient evaluation}

In the clinical outcome evaluation the following endpoints were considered: subjective IKDC score and level of sports activity, as evaluated with the Tegner activity score at 12 months of follow-up (6), and time (days) elapsed between surgery and clinical discharge. Discharge from the rehabilitation center was subject to fulfillment of all the relevant clinical and functional criteria (resolution of knee pain or swelling, recovery of ROM, muscle strength, and previous level of daily living activities or sport-specific activity). Both quantitative and qualitative variables for each patient were considered for correlation, divided into clinical background (patient-related) characteristics and post-surgical and rehabilitation management (rehabilitation-related) variables.

Among the clinical background characteristics, the following data were collected during the first postoperative visit: gender, age, weight, pre-injury Tegner activity score, sport practiced at professional or amateur level, mechanism of injury (contact or non-contact), presence of associated knee lesions, type of graft used, use of postoperative bracing, pain level (NRS 1$10)$, swelling (0-3 scale) and ROM at first postoperative visit, and days between surgery and beginning of rehabilitation.

The post-surgical and rehabilitation management variables included: number of rehabilitation sessions, number of gym sessions, number of pool sessions, number of OFR sessions, OFR sessions as a percentage of the total rehabilitation sessions (\%OFR), days between surgery and first knee IT, and maximum peak force (side-to-side difference) for knee extensors and knee flexor muscles at the first IT. The test was performed using an isokinetic dynamometer (Genu3; Easytech, Florence, Italy).

\section{Data analysis}

Statistical analysis was performed using Stata 12.1 (Statacorp Texas USA).

The data are presented as mean \pm standard deviation or median and relative range, as appropriate. Paired and unpaired t-tests were used to evaluate differences between quantitative variables. The linear regression model was used to identify possible relationships between clinical background and management variables and the IKDC score at one year of follow-up. The results were expressed as $\beta$ coefficients, with confidence intervals at $95 \%(95 \% \mathrm{CI})$ using univariate analysis. The relationship between different variables and the time between surgery and clinical discharge was analyzed with the Kaplan-Meier model. The number of days between surgery and clinical discharge was taken as the time variable, and the discharge itself as the event. The log-rank test was used to identify the statistically significant variables. They were further analyzed with the Cox regression model to understand which of them might independently affect, positively or negatively, the time between surgery and clinical discharge. The results were presented as Hazard ratio and $95 \% \mathrm{CI}$. All correlations were considered significant at $\mathrm{p}<0.05$.

\section{Results}

The mean IKDC subjective score at final evaluation was 91.2 \pm 11.9 (range, 32.2-100) and the mean Tegner activity score was 7.0 \pm 2.0 (range, 3-10).

As regards the post-surgical management, patients completed a mean number of $56 \pm 4$ (12-255) rehabilitation sessions: $36 \pm 2$ (range, 6-147) in the gym, 10 \pm 7 (range, 2-46) in the pool, and 10 \pm 9 (range, 0-62) OFR sessions. On average, OFR sessions accounted for $16.7 \pm 9 \%$ of the total (\%OFR range, $0-40)$. Patients were able to walk without crutches at a mean of $29.6 \pm 9.5$ days after surgery; they were able to run on a treadmill after $81.4 \pm 3$ days, and start the OFR after $100.5 \pm 38.5$ days. The first knee IT was performed $97.5 \pm 44.0$ days after surgery, and showed that $53 \%$ of the patients had a significant $(>20 \%)$ extensor strength deficit, whereas a significant flexor strength deficit was detected in $36 \%$, with a mean side-to-side deficit of maximal peak torque at $90^{\circ}$ that was higher for extensor (18.8\%, range 0-52) than flexor muscles $(12.5 \%$, range $0-72)$. The patients were discharged from the rehabilitation center $173 \pm 51$ (range, 84-362) days after surgery, and $161 \pm 53$ (range, 77-357) days after the start of the rehabilitation program. 


\section{oints}

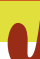

Further analysis was performed to evaluate factors influencing the IKDC score at final evaluation. Among the clinical background factors, a significantly higher IKDC subjective score was found at final evaluation in patients younger than 20 years and in patients with a higher pre-injury Tegner score. Significantly lower IKDC subjective scores were recorded by patients presenting with a concomitant combined collateral ligament (MCL or LCL) injury and by those who were prescribed a knee brace postoperatively. These patients also had a lower mean maximum knee flexion angle at the first postoperative consultation (57.5 vs 76.6, $\mathrm{p}=0.003)$, and also a higher mean knee flexor strength deficit at the first IT $(20.2 \%$ vs $11.1 \%, \mathrm{p}=0.04)$.

As regards the management variables correlated with the final IKDC score, the patients recording a higher $\%$ OFR ( i.e. $>15 \%$ ) were found to have a higher final IKDC score than those with a lower \%OFR. Two parameters recorded at the first IT for muscle strength assessment were also found to be correlated with the IKDC score: both knee extensor and knee flexor strength deficit $>20 \%$ were related to a lower final score (Tab. 2).

The discharge time was found to be correlated with three preoperative variables: practicing sport at a professional level (Fig. 1) and being under 20 years of age (Fig. 2) positively influenced the discharge time (Fig. 1), which on the other hand was negatively correlated with the presence of concomitant combined posterolateral corner (PLC) or posteromedial corner (PMC) tears (Fig. 3, Tab. 3). Patients in the professional level sport group walked without crutches after 21 days, versus 30 days in the other group ( $\mathrm{p}=0.08)$, and they were able to run on a treadmill at a mean of 53 days after surgery, versus 83 days in the amateur group $(\mathrm{p}=0.06)$.

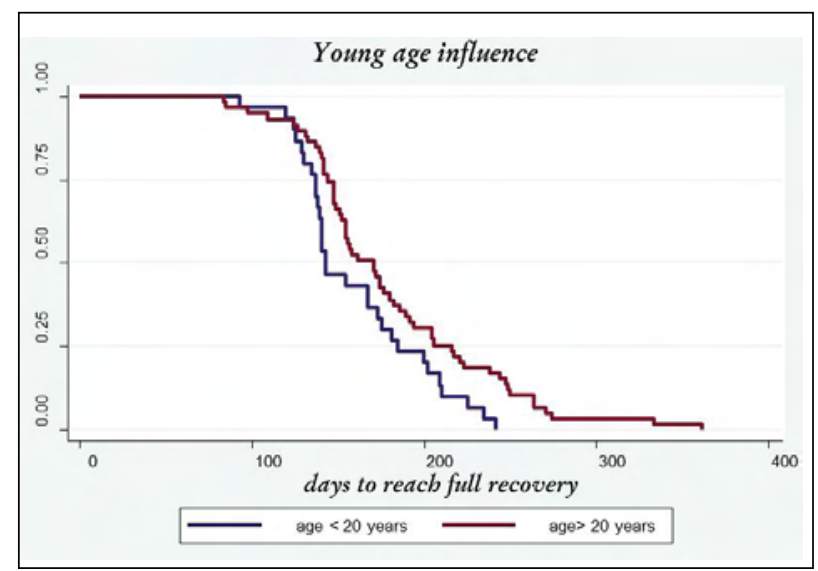

Fig. 1. The influence of young age on clinical discharge rate over time. Patients younger than 20 years were discharged earlier from the rehabilitation center.

Patients with combined PLC/PMC lesions needed 58 days more (154 vs 97, $\mathrm{p}=0.0009)$ before starting the OFR program, despite doing the same overall number of rehabilitation sessions and the same number of OFR sessions, and despite not showing significant differences in knee muscle strength parameters at the first IT.

Finally, a correlation was found between IKDC subjective score at 12 months of follow-up and discharge time. For each day of delay there was a decrease in the score $(\beta$ coefficient $=-0.05)$ : patients discharged before the $150^{\text {th }}$ day showed better results than those who were discharged later (94.5 us 89.1, respectively, $\mathrm{p}=0.03$ ).

Furthermore, taking the median number of days at the beginning of OFR as the cut-off ( $94 v s 88$, respectively, $\mathrm{p}=0.01$ ), patients starting OFR earlier were found to have a six-point higher IKDC score at 12 months' follow-up $(\mathrm{p}<0.005)$.

Table 2. Correlation between patient-related and rehabilitation-related variables and final clinical outcome, evaluated using the IKDC subjective score.

\begin{tabular}{llll}
\hline Variables & $\boldsymbol{\beta}$ Coefficient & $\mathbf{9 5 \%}$ C.I. & $\mathbf{p}$ \\
\hline Combined MCL/LCL injury (yes vs no) & -7.15 & $-13.3-0.96$ & 0.02 \\
Knee bracing (yes $v$ no) & -5.34 & $-10.3-0.31$ & 0.03 \\
Tegner pre-injury & 1.69 & $0.26-3.11$ & 0.02 \\
Age $(\leq 20$ yrs $v s>$ yrs) & 7.09 & $1.9-12.2$ & 0.007 \\
Gender (female $v$ s male) & 2.67 & $-3.00-8.34$ & 0.35 \\
Knee extensor strength deficit $(>20 \%$ vs $<20 \%)$ & -6.69 & $-11.3-1.99$ & 0.006 \\
Knee flexor strength deficit $(>20 \%$ vs $<20 \%)$ & -7.98 & $-12.7-3.20$ & 0.001 \\
$\%$ OFR $(<15 \%$ v $>15 \%)$ & -6.17 & $-11.2-1.12$ & 0.017 \\
\hline
\end{tabular}




\section{Discussion}

Even though ACLR is extensively investigated in the literature $(7,8)$, only a few studies have investigated preoperative and rehabilitation factors as predictors of outcome (in terms of clinical recovery) and of the

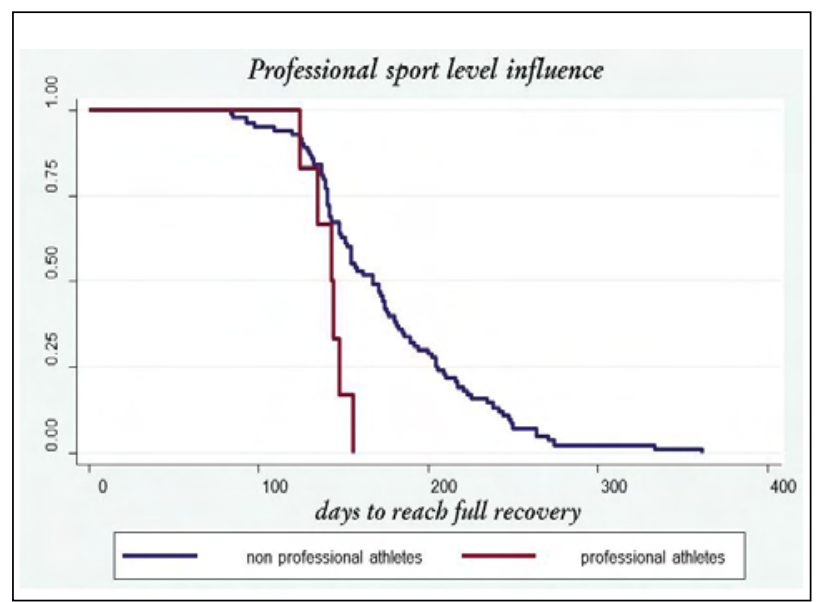

Fig. 2. The influence of the professional level sports activity on discharge rate over time. Professional athletes needed significantly less time to achieve a full recovery.

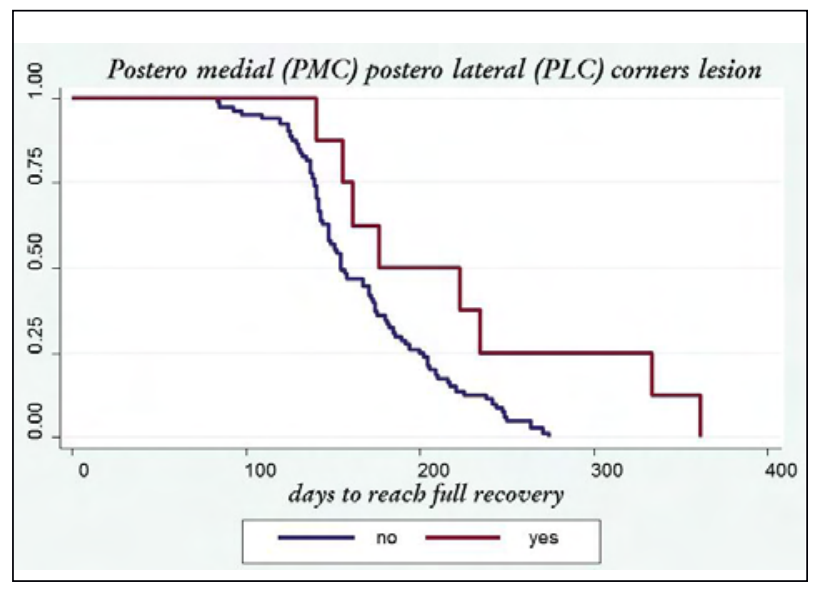

Fig. 3. The influence of PMC/PLC combined injury on clinical discharge rate over time. Patients with concurrent capsular lesions were discharged from the rehabilitation center later. time needed for recovery. In this series, several preoperative and management variables were found to be important factors influencing patients' final outcome and recovery time after ACLR. Four preoperative factors were found to be predictors of clinical outcome (age, presence of MCL/LCL injury, use of postoperative knee bracing, and pre-injury Tegner activity level) and three were independent predictors of recovery time (age, concomitant PMC/PLC injury and practicing sport at professional level). Concerning rehabilitation and management factors, a positive correlation was found with the percentage of OFR sessions, whereas flexor and extensor strength deficits $>20 \%$ at the first IT evaluation were related to inferior clinical results at one year.

In more detail, a lower functional score and a longer rehabilitation time was observed in patients who had multi-ligament injuries, took longer to start the OFR program, and thus showed a delayed functional recovery. This might be ascribed to the intrinsic complexity of multi-ligament injuries; indeed, such injuries may require additional procedures with ACLR (9), that might cause increased pain and symptoms, and require longer immobilization during the early postoperative phases (10).

This hypothesis seems to be confirmed by the reduced mean maximum knee flexion angle and increased mean knee flexor strength deficit recorded at the first postoperative consultation and at the first IT, respectively. These findings in this group of patients are in contrast to those of Spindler et al. (11), who did not find any difference in the clinical outcomes of patients with a collateral ligament injury concurrent with ACL damage at six years of follow-up. However, it is possible that the differences we found at short-term follow-up might decrease at a longer follow-up. Further prospective studies are required to analyze specifically and clarify exactly which factors affect the result: the longer immobilization that delays recovery of full ROM, or the additional instability (12). The complexity of concomitant lesions may also explain the poo-

Table 3. Correlation between patient and rehabilitation variables and clinical discharge time.

\begin{tabular}{llll}
\hline Variables & Hazard Ratio & $\mathbf{9 5 \%}$ C.I. & p \\
\hline Age $(\leq 20$ yrs $v s>20$ yrs) & 1.41 & $1.06-1.41$ & 0.02 \\
Professional athlete (yes $v$ no) & 2.98 & $1.24-7.12$ & 0.01 \\
PMC/PLC lesion (yes $v$ no) & 0.38 & $0.16-0.89$ & 0.02 \\
Gender (female $v$ s male) & 1.41 & $0.85-2.34$ & 0.18 \\
\hline
\end{tabular}


rer results we found in patients with postoperative knee bracing. In fact, in these patients the negative influence of bracing on outcome is probably due to the use of braces for more severe injuries $(60 \%$ of patients with concomitant collateral ligament injury $v s$ $40 \%$ of isolated ACL damage wore a postoperative knee brace); instead, in patients undergoing isolated ACLRs the IKDC score did not differ significantly according to whether patients did or did not wear a postoperative knee brace, similarly to what is already reported in the literature (13).

Age is another key factor influencing ACLR results: younger patients $(\leq 20$ years) showed both higher IKDC scores and a faster return to activities. As other studies previously demonstrated, age might be a relevant predictive factor for outcome after ACLR, possibly related to the intrinsic biological characteristics of younger people (11). Moreover, the younger patients also had a higher pre-injury activity level (Tegner 8.3 vs $7.3, \mathrm{p}=0.005)$ and a higher $\%$ OFR $(20 \%$ vs $15 \%$, $\mathrm{p}=0.02)$, even though the difference in the total number of OFR sessions was not statistically significant, and these factors might have contributed to their better outcome.

Patients with higher Tegner sport activity levels also recorded a higher IKDC score at the final evaluation. Previous findings by De Valk et al. support this correlation, which might be due to several factors (2). Patients with a higher Tegner score probably have better clinical and functional conditions prior to surgery; also, psychological factors might mean that they show better compliance during functional recovery phases. Moreover, the professional athletes reached were discharged earlier than the amateur and recreational athletes. Since sport is these patients' primary occupation, and it is particularly important for them to be back on the field as soon as possible, these patients are able to dedicate much more time to rehabilitation than recreational athletes can. Furthermore, the costs of rehabilitation might limit the compliance of the latter. This is confirmed by the fact that the professional group had a higher number of total rehabilitation (122 vs 55; $\mathrm{p}<0.00001)$ and $\mathrm{OFR}$ sessions (25.2 vs 9.6; $\mathrm{p}=0.0005)$ than the non-professional group, despite the shorter time-frame. Thus, more intensive rehabilitation might explain the faster recovery in this group, thus confirming previous findings after knee surgery by Della Villa et al., who reported a faster return to the team and to competition for professionals $v$ s amateurs in a cohort of 50 football players (14). A tendency towards faster progression in the early rehabilitation phases was also observed in this group: the professionals were able to walk without crutches and to run on a treadmill much sooner than the other patients.

With regard to rehabilitation and management factors, knee muscle strength deficit appeared to be a key factor in our analysis. The literature showed how preoperative knee extensor strength deficit can be a predictor of ACLR clinical outcome at six months of follow-up (15), and thus rehabilitation should aim at achieving muscle strength recovery. In particular, our findings confirmed the importance of muscle strength and suggested that a proper recovery of limb-to-limb symmetry should be reached as soon as possible, since an extensor deficit $>20 \%$ at first IT postoperatively was associated with lower scores at one year. Knee flexor strength was correlated with the IKDC subjective score at 12 months as well. Even though a knee flexor deficit $>20 \%$ at the first IT was less common than an extensor deficit, this finding still significantly affected the outcome in our series. Continuous evaluation of strength deficit by IT during the rehabilitation process would allow imbalances to be detected and corrected through specific interventions. Our findings show that it is important to achieve a complete recovery of function and strength before allowing unrestricted sports activity, partially because hamstring strength deficit has been identified as a factor contributing to the risk of re-injury after ACLR (16).

Finally, the proportion of OFR sessions performed influenced the functional result. Since the progressive resumption of the sport-specific gesture is the basic aim of the OFR concept (5), this finding should be considered relevant in the planning of postoperative treatment: the \% OFR could be increased in highly specialized centers to improve the functional result. In fact, patients who started OFR earlier had a faster recovery and a better outcome at one year.

Other factors such as gender, weight, mechanism of injury, type of graft used, pain, effusion and days between surgery and enrolment were not significantly correlated with outcome in our series. Some of these aspects are consistent with previous findings: most Authors reported no differences using different grafts or surgical techniques $(8,17,18)$, while the role of gender is more controversial (7), but we cannot exclude 
that future studies on larger cohorts of patients might also reveal an influence of these factors on the results of ACLR. In fact, limitations of the present study are the limited number of patients, which restricts the possibility of performing sub-analyses and detecting confounding factors, and the short-term evaluation, which prevents us from showing whether the observed variables might also influence the long-term clinical outcome. Another weakness is the lack of preoperative IT data. However, this study highlighted the importance of several factors, both patient-related and rehabilitation-related, which were found to play a role and therefore warrant investigation in future robust studies, in order to improve the management of patients undergoing ACLR.

In conclusion, ACLR and rehabilitation have a high success rate. Nonetheless, personal, surgical and rehabilitation factors should be considered in order to optimize patient management and eventually improve the final outcome and prevent re-ruptures. In fact, sports activity level, multi-ligament injuries, and muscle strength status were found to influence clinical outcome in this study. Future studies with larger numbers of patients and stronger designs, aimed at evaluating the factors leading to better outcomes, will help to improve ACLR treatment and enable patients to achieve a safe recovery and a faster return to sports activities.

\section{References}

1. Gomoll AH, Filardo G, Almqvist FK, et al. Surgical treatment for early osteoarthritis. Part II: allografts and concurrent procedures. Knee Surg Sports Traumatol Arthrosc. 2012; 20:468-486.

2. de Valk EJ, Moen MH, Winters M, et al. Preoperative patient and injury factors of successful rehabilitation after anterior cruciate ligament reconstruction with single-bundle techniques. Arthroscopy. 2013;29:1879-1895.

3. Barber-Westin SD, Noyes FR. Factors used to determine return to unrestricted sports activities after anterior cruciate ligament reconstruction. Arthroscopy. 2011;27:1697-1705.

4. Petersen W, Zantop T. Return to play following ACL reconstruction: survey among experienced arthroscopic surgeons (AGA instructors). Arch Orthop Trauma Surg. 2013;133: 969-977.
5. Della Villa S, Boldrini L, Ricci M, et al. Clinical outcomes and return-to-sports participation of 50 soccer players after anterior cruciate ligament reconstruction through a sport-specific rehabilitation protocol. Sports Health. 2012;4:17-24.

6. van Meer BL, Meuffels DE, Vissers MM, et al. Knee injury and Osteoarthritis Outcome Score or International Knee Documentation Committee Subjective Knee Form: which questionnaire is most useful to monitor patients with an anterior cruciate ligament rupture in the short term? Arthroscopy. 2013;29:701-715.

7. Ardern CL, Taylor NF, Feller JA, et al. Fifty-five per cent return to competitive sport following anterior cruciate ligament reconstruction surgery: an updated systematic review and meta-analysis including aspects of physical functioning and contextual factors. Br J Sports Med. 2014;48:1543-1552.

8. Cvetanovich GL, Mascarenhas R, Saccomanno MF, et al. Hamstring autograft versus soft-tissue allograft in anterior cruciate ligament reconstruction: a systematic review and meta-analysis of randomized controlled trials. Arthroscopy. 2014;30:1616-1624.

9. Bonanzinga T, Zaffagnini S, Grassi A, et al. Management of combined anterior cruciate ligament-posterolateral corner tears: a systematic review. Am J Sports Med. 2014;42:14961503.

10. Flandry F, Baker CL, Jr, Jacobson KE, et al. Evaluation and treatment of acute and chronic injuries to the capsular ligaments of the knee. Instr Course Lect. 2009;58:397-421.

11. Spindler KP, Huston LJ, Wright RW, et al. The prognosis and predictors of sports function and activity at minimum 6 years after anterior cruciate ligament reconstruction: a population cohort study. Am J Sports Med. 2011;39:348-359.

12. Signorelli C, Bonanzinga T, Lopomo N, et al. Do pre-operative knee laxity values influence post-operative ones after anterior cruciate ligament reconstruction? Scand J Med Sci Sports. 2013;23:e219-224.

13. Lobb R, Tumilty S, Claydon LS. A review of systematic reviews on anterior cruciate ligament reconstruction rehabilitation. Phys Ther Sport. 2012;13:270-278.

14. Della Villa S, Kon E, Filardo G, et al. Does intensive rehabilitation permit early return to sport without compromising the clinical outcome after arthroscopic autologous chondrocyte implantation in highly competitive athletes? Am J Sports Med. 2010;38:68-77.

15. Logerstedt D, Lynch A, Axe MJ, et al. Pre-operative quadriceps strength predicts IKDC2000 scores 6 months after anterior cruciate ligament reconstruction. Knee. 2013;20:208-212.

16. Hewett TE, Di Stasi SL, Myer GD. Current concepts for injury prevention in athletes after anterior cruciate ligament reconstruction. Am J Sports Med. 2013;41:216-224.

17. Mascarenhas R, Erickson BJ, Sayegh ET, et al. Is there a higher failure rate of allografts compared with autografts in anterior cruciate ligament reconstruction: a systematic review of overlapping meta-analyses. Arthroscopy. 2015;31:364-372.

18. Andernord D, Bjornsson H, Petzold M, et al. Surgical predictors of early revision surgery after anterior cruciate ligament reconstruction: results from the Swedish National Knee Ligament Register on 13,102 patients. Am J Sports Med. 2014,42:1574-1582. 